\title{
BIENESTAR PSICOLÓGICO: ÁMBITO DE ACTUACIÓN PARA EL PSICÓLOGO UNADISTA
}

\author{
Jhon Fredy Bustos Ruiz \\ Correo electrónico: jhon.bustos@unad.edu.co \\ ORCID ID: 0000-0003-0118-2056 \\ Mabel Goretty Chala Trujillo \\ Correo electrónico: mabel.chala@unad.edu.co \\ ORCID ID: 0000-0001-7183-1723
}

Abril de 2017

Palabras clave: Bienestar psicológico, psicología, educación infantil, potenciación, acción psicosocial.

Solidez curricular, gestión formativa e investigación son elementos esenciales en la ecuación que determina la calidad en la educación universitaria; y cada uno alcanza su mayor potencia en tanto se mantiene en interdependencia con los demás. En dicha lógica, este documento es un aporte para que esa interrelación investigación-currículoacademia en el caso del programa de psicología de la UNAD se fortalezca. Se trata de presentar una reflexión derivada de procesos investigativos adelantados en el grupo de investigación "Psicología, Desarrollo Emocional y Educación", adscrito al programa de Psicología, según la cual la categoría teórica Bienestar Psicológico puede constituirse en un ámbito de actuación interesante para el psicólogo de la UNAD.

El programa de psicología de la UNAD, con 23 años de trayectoria en Colombia, en su documento condiciones de calidad del programa de psicología con fines de Renovación de registro calificado elaborado de conformidad con el decreto 1295 (2013) indica que "se fundamenta desde una perspectiva social comunitaria, la cual se constituye en su impronta. Sienta sus fundamentos epistemológicos y teóricos en la psicología clásica, así como en los desarrollos contemporáneos de las epistemologías latinoamericanas y de la psicología crítica, comprendiendo la acción psicosocial como un conjunto de estrategias que: (a) Posibilita el reconocimiento de individuos y comunidades para fortalecer su capacidad de autogestión (b) Genera estilos de relación para la convivencia saludable y (c) Propende por el desarrollo de proyectos colectivos en aras de mejorar la calidad de vida" $56 \mathrm{p}$. Desde esta perspectiva, se propende por la formación de un Psicólogo conocedor de los fundamentos científicos básicos de la Psicología, capaz de comprender, interpretar y analizar el comportamiento humano; con las destrezas básicas para evaluar y actuar en el ámbito individual, grupal y comunitario, de manera ética, con sentido crítico e investigativo y compromiso social.

Por lo anterior, el "Psicólogo Unadista en su formación profesional, desarrolla competencias para diseñar, implementar y asesorar intervenciones psicosociales encaminadas a:

1. Crear y desarrollar servicios alternativos de protección, salud, educación y rehabilitación, cuyo diseño, provisión y expansión estén basados en la autogestión y/o la participación de las comunidades.

2. Desarrollar e incrementar la capacidad de asociación y autogestión de los grupos humanos. 
3. Fortalecer la estrategia de trabajo en red en las comunidades con el fin de promover el desarrollo psicosocial de colectividades de diferente procedencia étnica, cultural y social.

4. Diseñar y evaluar procesos e impacto de programas, proyectos y servicios de salud, educación, rehabilitación, protección, convivencia y desarrollo de grupos humanos.

5. Producir conocimiento e intervenir los procesos psicosociales de organización humana para el trabajo; especialmente en el contexto de condiciones asociativas. Así mismo posibilitar escenarios en los cuales se transformen las pautas relacionales en los entornos organizacionales, potencializando las capacidades del Talento Humano existente dentro de ellas.

6. Diseñar e implementar proyectos de investigación intra e interdisciplinarios para la generación de nuevos conocimientos que contribuyan a la disciplina y den respuesta a las necesidades y procesos psicosociales en los diferentes campos de la disciplina a nivel local y global" $57 \mathrm{p}$.

Para orientar este proceso formativo, el diseño curricular del programa de Psicología de la UNAD está concebido en la lógica de Núcleos Problémicos (NP). Definidos desde el documento Redes Académicas Diseño Curricular Programa de Psicología (2014), como "unidad integradora que posibilita el pensar en procesos de investigación y formación, alrededor de objetos de transformación. EI NP sugiere estrategias metodológicas que garanticen la síntesis creativa entre la teoría y la práctica; permite un diseño curricular dinámico, coherente y problematizador, que redefine la concepción de estructuras "asignaturistas" y busca propiciar un aprendizaje bajo el esquema de procesos lógicos, que incitan al estudiante a enfrentarse a problemas reales movilizando sus recursos cognoscitivos, axiológicos y contextuales" $7 \mathrm{p}$.

Este mismo documento indica que curricularmente, el programa de Psicología está diseñado desde cinco NP y un Núcleo Integrador Problémico (NIP) denominado Conocimiento Psicosocial y Praxis. Desde este NIP, se entiende que “...la formación de psicólogos requiere abordajes teóricos y metodológicos que trascienden las posturas tradicionales, las cuales implican hacer una transición de modelos clínicos a modelos que incluyan la educación y la prevención" 48 p. Por tanto, “...la responsabilidad social del Programa de Psicología de la UNAD se dimensiona al indicar que en él se forman los profesionales de la Psicología que asumen el reto de contribuir a la transformación de la realidad que les es propia, que se forman como psicólogos desde sus lugares de origen y que ejercen su profesión en el lugar que se requiere, el lugar donde se necesitan, el lugar donde es necesaria la acción de un profesional: su lugar de origen, su municipio, su vereda, su barrio... [De esta manera, se propicia] una mirada integradora de realidades, situaciones, circunstancias y condiciones, con el objetivo de formar con calidad y calidez, liga lo académico formativo a una cultura de calidad, y lo comunitario a una sensibilidad social humanizante..." $51 \mathrm{p}$.

Es justamente desde el NIP que el Bienestar Psicológico, como ámbito de formación y actuación del psicólogo Unadista, encuentra espacio para insertarse al currículo. Ello implica pensar en el BP como categoría teórica que debe estar incluida dentro del plan de estudios del programa de una manera más precisa, de forma que se garantice su abordaje conceptual y propicie el desarrollo de las competencias necesarias a un modelo de prevención. La idea de incorporar el BP como ámbito de actuación para el psicólogo Unadista tiene que ver con el reconocimiento de los hallazgos que la 
investigación en esta línea ha ido arrojando, y muy especialmente en las conclusiones que hasta el momento se han ido formulando desde el grupo de investigación Psicología, Desarrollo Emocional y Educación.

Desde el marco general se ha establecido, por ejemplo, que el BP es “...un importante predictor de la salud física, de la longevidad, de las relaciones interpersonales y del funcionamiento psicológico óptimo de las personas" (Diener y Biswas - Diener, 2008; Citado por Solano, 2009) .... Adicionalmente, son amplias las posibilidades de un buen "pronóstico" para procesos de intervención fundamentados en el bienestar psicológico; ya que muchas de "las intervenciones psicológicas, en su fase exploratoria, dan cuenta de que el bienestar se puede modificar y que es posible elevar la capacidad hedónica de las personas mediante la actividad intencional" (Solano, 2009).

En el contexto más específico, el grupo de investigación "Psicología, Desarrollo Emocional y Educación", adscrito al programa de Psicología, ha desarrollado dos proyectos de investigación en el tema de felicidad y bienestar psicológico. Para los fines de este documento se trae a colación el proyecto denominado "Movilizaciones psicoafectivas derivadas de la implementación de un modelo psicoeducativo fundamentado en el concepto de bienestar psicológico, en los niños escolares de Bogotá, Pasto y Neiva", desarrollado desde finales de 2015. En dicha investigación se pretendió "Reconocer las movilizaciones psico-afectivas derivadas de la implementación de un modelo psicoeducativo fundamentado en el concepto de bienestar psicológico, en los niños escolares de Bogotá, Pasto y Neiva". Este proceso investigativo tuvo como eje central a los niños y niñas, no obstante, incorporó también la participación de sus docentes y padres de familia.

Tal como se enuncia en su título, la investigación se fundamentó en el modelo multidimensional de Carol Ryff (1989). De acuerdo con este modelo de seis dimensiones el BP de un individuo en cualquier etapa del ciclo vital está dado por el grado de desarrollo de cada una de estas dimensiones: Autonomía, Auto aceptación, Relaciones positivas con otras personas, Dominio del entorno, Propósito de vida u objetivos vitales y Crecimiento personal. A continuación, una breve alusión a cada una de las dimensiones del BP desde Carol Ryff:

Autonomía. Para sostener su individualidad en diferentes contextos sociales, las personas necesitan asentarse en sus propias convicciones (autodeterminación) y mantener su independencia y autoridad personal (Ryff y Keyes, 1995). Las personas con autonomía son capaces de resistir en mayor medida la presión social y auto-regulan mejor su comportamiento (Ryff y Singer, 2002).

Auto-aceptación es uno de los criterios centrales del bienestar. Las personas intentan sentirse bien consigo mismas incluso siendo conscientes de sus propias limitaciones. Tener actitudes positivas hacia uno mismo es una característica fundamental del funcionamiento psicológico positivo (Keyes et al., 2002).

Relaciones positivas con otras personas (Erikson, 1996; Ryff y Singer, 1998). La gente necesita mantener relaciones sociales estables y tener amigos en los que pueda confiar. La capacidad para amar es un componente fundamental del bienestar (Allardt, 1996) y consiguientemente de la salud mental (Ryff, 1989b). De hecho, numerosas investigaciones realizadas en las dos últimas décadas (Berkman, 1995; Davis, Morris y Kraus, 1998; House, Landis, y Umberson, 1988) señalan que el aislamiento social, la soledad, y la pérdida de apoyo social están firmemente relacionadas con el riesgo de padecer una enfermedad y reducen el tiempo de vida. 
Dominio del entorno, es decir, la habilidad personal para elegir o crear entornos favorables para satisfacer los deseos y necesidades propias es otra de las características del funcionamiento positivo. Las personas con un alto dominio del entorno poseen una mayor sensación de control sobre el mundo y se sienten capaces de influir sobre el contexto que les rodea.

Propósito en la vida. Las personas necesitan marcarse metas, definir una serie de objetivos que les permitan dotar a su vida de un cierto sentido. Necesitan, por tanto, tener un propósito en la vida, objetivos vitales claros que les permitan orientar sus acciones cotidianas con significado visión de futuro.

Crecimiento personal. El empeño por desarrollar sus potencialidades, por seguir creciendo como persona y llevar al máximo sus capacidades (Keyes et al, 2002).

Ahora bien, como parte del análisis de resultados de la investigación se hicieron notorias algunas situaciones que ponen de manifiesto el espacio, la conveniencia y la importanciasde que desde la perspectiva del bienestar psicológico se tenga una línea de acción para el ejercicio del rol del psicólogo con énfasis social comunitario de la UNAD. A continuación se comparten esas situaciones y reflexiones más pertinentes.

Desde el discurso de los niños y las niñas se puede reconocer que, a su edad, si bien, no tienen una claridad siquiera mediana sobre el BP, la cotidianidad que expresan expone limitantes en su proceso de construcción de ese BP. Los niños tienen sueños y proyectos para sus vidas. Los niños se sienten movidos a tomar sus propias decisiones. Los niños reconocen hasta cierto punto sus fortalezas y debilidades. Los niños avanzan en la construcción de relaciones con otros. Los niños quieren salir de sus casas y conocer el mundo. Infortunadamente no en todos los casos eso que descubre el discurso de los niños y las niñas está en la línea adecuada para concretarse en niveles deseables de autonomía, relaciones positivas, autoaceptación, crecimiento personal, dominio del entorno y propósito en la vida. Esto implica que se requieren procesos estructurados y fundamentados que propicien las condiciones necesarias para consolidar una ruta hacia el BP.

A veces las circunstancias de vulnerabilidad social es la principal limitante, pero aún en tales casos es que el enfoque de BP puede resultar más necesario.

En el caso de los padres su discurso revela interés y compromiso, aunque no siempre pertinencia y eficacia. Incluso algunas acciones que pudieran ser cuestionables son defendidas y justificadas desde lo emocional más no desde lo técnico. Una idea de algunos padres de familia que llamó poderosamente la atención es aquella según la cual su compromiso es tal que en función de sus roles han dejado de lado su propio bienestar en favor de garantizar el bienestar de sus hijos. Esto desde luego es cuestionable, dado que, desde la perspectiva del bienestar subjetivo y la felicidad, sólo se puede contribuir a la felicidad de otro cuando uno mismo es feliz. En este sentido, dejar de lado el propio desarrollo, no necesariamente propicia el desarrollo de los hijos.

Lo que esto revela a los investigadores es justamente la carencia de una estructura conceptual y metodológica que desde la comprensión de los roles paterno y materno permitan procesos educativos que favorezcan el BP.

En cuanto a los docentes, se encuentra que perciben una escasa articulación en la corresponsabilidad de padres de familia y docentes en aras de adelantar sus roles de educadores de niños y niñas. Los docentes perciben la complejidad de los contextos 
actuales, identificando que los niños y las niñas deben enfrentar situaciones difíciles en su entorno inmediato, que se constituyen en factores de riesgo frente a las relaciones que establecen con otras personas. Los docentes, a pesar de reconocer grandes limitaciones en su rol, tratan de jugar un papel importante en la formación, no sólo académica, sino también personal, de sus estudiantes, ya que tienen claro que son un referente importante y los niños comparten un espacio que puede aprovecharse para lograr generar bienestar psicológico en ellos. Sin embargo, no encuentran la suficiente adherencia de los padres al proceso formativo de sus hijos, pues los docentes perciben que los padres consideran que escuela y familia no necesariamente deben interactuar para favorecer la educación de los niños y las niñas. Lo que debe agregarse es que en el ámbito educativo tampoco hay una estructura que facilite procesos en función del BP; en parte por la complejidad de la realidad misma, en parte por la carencia de competencias del personal docente en lo relativo a BP y en parte porque no hay una línea de acción reconocida sobre BP.

Tanto las manifestaciones de los padres como de los docentes evidencian necesidad de potenciar sus propias habilidades y características en el desarrollo del SER, pues si bien, cada uno de ellos reconocen algunas de sus debilidades, sus fortalezas y la influencia que ejercen en sus hijos o estudiantes, sus experiencias vitales los llevan a generar un estilo de vida particular que no necesariamente conduce al BP, por lo menos no desde las categorías estudiadas.

A este respecto, Espinosa, M. y Ochaíta, E. (2000) indican que "La escuela debe propiciar la construcción activa del conocimiento por parte de los niños, no se trata sólo de memorizar contenidos sino de integrarlos realmente en esquemas cognitivos. Debe también propiciar la participación y el aprendizaje de hábitos democráticos de convivencia. Y esto no sólo de manera formal, con la existencia de los alumnos en los consejos escolares, sino con el respeto por sus ideas y propiciando el aprendizaje de la toma de decisiones colectivas..." $9 \mathrm{p}$.

Acorde con la postura Piagetiana sobre el desarrollo cognoscitivo, las mismas autoras expresan, la manifestación de las necesidades básicas en la etapa de las operaciones concretas (6 a 11/12 años), que se resume en el siguiente cuadro:

\begin{tabular}{|l|l|l|}
\hline \multicolumn{2}{|c|}{ Salud Física } & \multicolumn{2}{c|}{ Autonomía } \\
\hline $\begin{array}{c}\text { Necesidades } \\
\text { biológicas }\end{array}$ & \multicolumn{1}{|c|}{ Necesidades sexuales } & $\begin{array}{c}\text { Necesidades psicológicas } \\
\text { y sociales }\end{array}$ \\
\hline $\begin{array}{l}\text { Alimentación } \\
\text { variada }\end{array}$ & Información adecuada & Educación escolar \\
\hline Sueño ordenado & $\begin{array}{l}\text { Conocimiento del propio cuerpo } \\
\text { y su desarrollo }\end{array}$ & Vínculos afectivos familiares \\
\hline Higiene & Curiosidad & $\begin{array}{l}\text { Participación familiar y } \\
\text { escolar }\end{array}$ \\
\hline Vivienda & Imitación & Relaciones con iguales \\
\hline
\end{tabular}




\begin{tabular}{|l|l|l|}
\hline Ejercicio físico & Autoestimulación & Ocio y tiempo libre \\
\hline $\begin{array}{l}\text { Actividades al aire } \\
\text { libre }\end{array}$ & Protección de riesgos & $\begin{array}{l}\text { Aprendizaje de reglas } \\
\text { y normas en los juegos }\end{array}$ \\
\hline
\end{tabular}

Tomado de: Espinosa, Ma. Angeles y Ochaíta, Esperanza. (2000). Necesidades infantiles y adolescentes y maltrato. Psykhe Vol. 9, No. 2, 3-15. Universidad Autónoma de Madrid. 9 p.

Esto implica que para propiciar el desarrollo y el bienestar psicológico de niños y niñas es relevante conocer sus necesidades y atenderlas, para lo cual, la familia juega un papel fundamental, como lo anota Montero (1989) citado por Vielma (2003), "Algunos estudios realizados por Montero (1989) demuestran el papel fundamental que cumple la familia como agente básico dentro del proceso de socialización y como fuente de aprendizaje en general que incluye desde los hábitos culturales hasta la organización cognitiva del individuo y la estructuración de su personalidad. Los resultados de sus estudios permiten dilucidar cómo, entre la familia y los miembros que la integran, se produce una condición de dependencia denominado Efecto de Primacía, a través del cual los padres influyen más acentuadamente en los nuevos hijos e hijas nacido(a)s dentro de ella que otros agentes socializadores coexistentes. Dicho efecto de primacía tenderá a manifestarse sobre lo(a)s hijo(a)s según sea la capacidad de control e influencia de los padres y de quienes hacen sus veces" $3 p$.

La misma autora indica que "Gracias al proceso de socialización, los individuos logran desarrollar la capacidad del aprendizaje social interiorizado o internalizando los elementos normativos implícitos en las prácticas sociales, en el discurso y en el quehacer cotidiano" $5 \mathrm{p}$. Esto significa que para propiciar el bienestar psicológico de niños y niñas los adultos involucrados en los procesos de socialización primaria y secundaria (padres y docentes) necesariamente deben propender por el bienestar psicológico propio, con el fin de que puedan constituirse en agentes favorecedores de la felicidad de los niños y las niñas.

Contribuir al desarrollo de la Autonomía, la Auto aceptación, las Relaciones positivas con otras personas, el Dominio del entorno, el Propósito de vida o el establecimiento de objetivos vitales y el Crecimiento personal en un niño o niña requiere de un adulto que de ejemplo, lo cual, a su vez exhorta consciencia y decisión de parte del adulto, esto es, determinación para buscar el bienestar propio y el desarrollo de las dimensiones que lo conforman de manera consistente, de tal forma que la búsqueda y vivencia del bienestar se evidencie en la vida cotidiana y a pesar de las vicisitudes.

Estos hallazgos evidencian necesidades de intervención psicosocial susceptibles de ser atendidos desde la lógica de la potenciación del BP, desde una o varias de las dimensiones que lo componen. Niños, padres y docentes revelan en sus discursos y prácticas cotidianas diferentes grados de acercamiento a condiciones deseables para el desarrollo de las dimensiones del BP. Esto implica la posibilidad de perfilar el ámbito de actuación para el psicólogo Unadista desde la promoción y la gestión del Bienestar Psicológico de las personas en los diferentes contextos, teniendo en cuenta:

1. El bienestar psicológico se refiere al funcionamiento positivo en la vida cotidiana. Incluye dimensiones psicológicas y sociales que se evidencian en conductas relacionadas con la salud en general y con las maneras de manejar los retos de 
la vida aprendiendo de ellos y profundizando en la experiencia de vivir con un sentido claro para cada persona.

2. Para generar en niños y niñas, procesos de socialización adecuados que se orienten a su bienestar psicológico, es crucial el apoyo de adultos que asuman su propio bienestar con la consciencia de que el ejemplo es el mejor mecanismo educador.

3. El favorecimiento del bienestar psicológico en cualquier etapa de la vida genera ambientes que contribuyen al desarrollo de las personas, el logro de metas, el sentido profundo de vivir, el desarrollo de virtudes y en últimas, el bienestar general de diferentes personas en diversos contextos.

4. Promover el desarrollo de las dimensiones del bienestar psicológico propuestas por Ryff (1989) como son la Autonomía, la Auto aceptación, las Relaciones positivas con otras personas, el Dominio del entorno, el Propósito de vida o el establecimiento de objetivos vitales y el Crecimiento personal, requiere la evolución de las características personales y el estilo de vida en cualquier etapa del ciclo vital.

5. El psicólogo, inclusive desde el rol de docente orientador, es el profesional competente para implementar y promover una línea de trabajo con estructura y sentido claros que desde el ámbito escolar potencie condiciones para el BP.

Estas ideas fundantes nos invitan a pensar en rutas de acción que puedan favorecer tanto la calidad del acompañamiento que los adultos pueden ofrecer a los niños y las niñas a su cargo, como la potenciación de los mismos adultos (padres y docentes), beneficiando el bienestar psicológico de adultos y niños para posibilitar la construcción de familias y entornos con personas más felices, lo cual puede llevarnos a incorporar elementos curriculares al programa de Psicología de la UNAD que conlleven a establecer líneas de acción para el trabajo de todos los actores académicos del programa y fortalecer el eje diferenciador del Psicólogo Unadista.

\section{Referencias:}

Ochaíta \& Espinosa. (1998) citado por Espinosa, M. y Ochaíta, E. (2000). Necesidades infantiles y adolescentes y maltrato. Psykhe Vol. 9, No. 2, 3-15. Universidad Autónoma de Madrid.

UNAD. Escuela de Ciencias Sociales, Artes y humanidades. (2013). Documento condiciones de calidad del programa de psicología con fines de renovación de registro calificado elaborado de conformidad con el decreto 1295.

UNAD. Escuela de Ciencias Sociales, Artes y humanidades. (2014). Redes Académicas Diseño Curricular Programa de Psicología.

Vielma, J. (2003). Estilos de crianza, Estilos educativos y Socialización: ¿Fuentes de Bienestar Psicológico?. Acción Pedagógica. Vol. 12, No. 1. Universidad de Los Andes. 